\title{
Dimensão econômica de sistemas de integração lavoura-pecuária
}

\author{
Geraldo Bueno Martha Júnior(1), Eliseu Alves ${ }^{(2)}$ e Elisio Contini(1)
}

(1)Embrapa Estudos e Capacitação, Parque Estação Biológica, Av. W3 Norte (final), CEP 70770-901 Brasília, DF. E-mail: geraldo.martha@embrapa.br, elisio.contini@embrapa.br (2)Embrapa Sede, Parque Estação Biológica, Av. W3 Norte (final), CEP 70770-901 Brasília, DF. E-mail: eliseu.alves@embrapa.br

\begin{abstract}
Resumo - Os sistemas de integração lavoura-pecuária (ILP) têm sido propostos como solução tecnológica para atender critérios de sustentabilidade, mas tal assertiva tem-se baseado principalmente em indicadores agronômicos e ambientais. Neste trabalho, explorou-se a perspectiva econômica da ILP. Os benefícios econômicos potenciais desses sistemas podem refletir-se em economia de escopo (diminuição do custo, em razão da produção de múltiplos produtos) ou nos efeitos de redução de risco pela diversificação. Além disso, eles podem envolver menor variabilidade produtiva e maior produtividade. A mensuração acurada das interações entre os componentes lavoura e animal (pastagem) é etapa-chave a ser perseguida. A tomada de decisão em prol de sistemas diversificados vis-à-vis sistemas especializados deve ser feita de acordo com os preços relativos. A ILP compete com sistemas especializados de pecuária, mas não apresenta taxas de retorno competitivas em comparação a sistemas especializados com soja. A elevada demanda por capital da ILP, particularmente para a aquisição de animais em recria para a engorda, explica as menores taxas de retorno da ILP e é vista como uma das principais restrições para a ampla adoção de sistemas mistos. O desenho de mecanismos de financiamento inovadores será essencial para promover e acelerar a adoção em larga escala da tecnologia.
\end{abstract}

Termos para indexação: análise de risco, diversificação, economia de escopo, pastagem degradada, pecuária de corte, soja.

\section{Economic dimension of integrated crop-livestock systems}

\begin{abstract}
Integrated crop-livestock systems (ICLS) have been proposed as a technology solution to meet sustainability criteria, but this claim has been based mainly on agronomic and environmental indicators. In the present study, the economic perspective of ICLS was explored. The potential economic benefits of these systems may be reflected in economies of scope (cost reduction associated with producing multiple outputs) or in the risk-reducing effects of diversification. Furthermore, they may involve lower yield variability and overall higher yields. The accurate measurement of interactions between crop and animal (pasture) components is a key step to be pursued. Decision making toward diversified vis-à-vis specialized agricultural systems must be made according to relative prices. ICLS out-compete specialized livestock systems but do not show competitive rates of return in comparison to specialized soybean systems. The high demand for capital in ICLS, particularly for the acquisition of growing animals for fattening, explains ICLS' lower return rates and is perceived as a major constraint on the widespread adoption of mixed systems. The design of innovative financing mechanisms will be essential to foster and speed large-scale adoption of the technology.
\end{abstract}

Index terms: risk analysis, diversification, economies of scope, degraded pasture, beef cattle, soybean.

\section{Introdução}

Os sistemas de integração lavoura-pecuária (ILP) figuram como opção interessante para assegurar a expansão da agropecuária, com baixa pressão sobre o avanço da fronteira agrícola, e estão de acordo com uma estratégia de baixo carbono (Gouvello, 2010; Herrero et al., 2010).

Aspectos econômicos de novas tecnologias $v i s-\grave{a}$-vis aquelas em uso na propriedade, incorporandose a influência de eventuais incentivos e do risco, são determinantes para a adoção dos diferentes sistemas de produção pelos produtores rurais. Pela perspectiva econômica, a pergunta relevante é se, dada uma oferta ambiental, os preços relativos de produtos e insumos levam à tomada de decisão para a especialização dos sistemas agropecuários ou para a sua diversificação por meio da integração lavoura-pecuária.

A maioria das propriedades agrícolas produz, no espaço e no tempo, mais de um produto. Entretanto, ao longo dos séculos, e de maneira bastante intensa a partir de meados do século XX, as propriedades agrícolas passaram a se especializar na produção de poucas commodities. Estatísticas do governo dos Estados Unidos mostraram que, em 1900, produzia-se, naquele país, a média de 5,1 commodities por fazenda. 
Em 1970, esse valor havia diminuído para 2,7 e, em 2000/2002, a quantidade média de commodities por fazenda era de 1,3. Garagorry et al. (2003) trabalharam com informações de 1.791 estabelecimentos rurais no Brasil e encontraram concentração nas duas classes de maior especialização, isto é, com um produto responsável por mais de $80 \%$ da receita ou com o produto mais importante respondendo por 50 a $80 \%$ da receita, e a segunda atividade por mais de $10 \%$.

Indivíduos e regiões se especializam de acordo com suas vantagens comparativas. Para um dado nível de risco, busca-se produzir um bem ou serviço a um custo de oportunidade menor do que outros produtores e, com isso, aumentar a rentabilidade do negócio. Com a especialização, as fazendas podem capturar mais facilmente economias de escalas para produtos específicos. Ademais, em termos regionais, a especialização permite o acúmulo de conhecimento coletivo em poucos assuntos. Assim, fica mais barato buscar informação e, frequentemente, é possível baixar o custo de produção.

$\mathrm{Na}$ última década, no entanto, o interesse por sistemas mistos de lavoura e pecuária tem aumentado, em particular pela pesquisa. Em grande parte, essas ações da pesquisa respondem à constatação de que sistemas especializados de produção de grãos têm sido associados à deterioração da qualidade física do solo (Ayarza et al., 1999), com maiores perdas de solo e água (Dedecek et al., 1986), maior incidência de pragas, doenças e invasoras (Vilela et al., 1999; Kluthcousky et al., 2003) e com alterações desfavoráveis na população de fungos micorrízicos do solo (Miranda et al., 2005).

Pela ótica econômica, é necessário que se quantifique como essas potenciais vantagens dos sistemas mistos, e outras - como a capacidade de mitigação do carbono da atmosfera pelo aumento no teor de matéria orgânica do solo, a maior eficiência de uso de insumos, e a redução da emissão de metano pelos animais em pastejo e, potencialmente, do risco de produção -, podem transformar-se em ganhos econômicos. Alguns exemplos de efeitos sinérgicos na integração lavoura-pecuária são apresentados na Tabela 1. Assim, quantificar adequadamente as interações entre os componentes pasto e lavoura, conferindo-lhes valores monetários, é passo prioritário para verificar o real desempenho econômico da ILP vis-à-vis o de sistemas especializados.

Conceitualmente, para medir a resposta econômica na ILP, seria necessária uma função custo que incorporasse os " $n$ " sistemas envolvidos e permitisse ajustes relevantes quanto aos efeitos de interações entre os componentes lavoura e pecuária. Embora a pesquisa agronômica em ILP tenha avançado substancialmente nas últimas décadas (Portes et al., 2000; Klutchcousky et al., 2003; Sulc \& Tracy, 2007; Maughan et al., 2009; Souza et al., 2010; Vilela et al., 2011), há necessidade de se explorar, em desenhos experimentais futuros, arranjos que permitam estimar, com clareza, a função custo de sistemas multiprodutos vis-à-vis a de sistemas especializados. Com esse procedimento, é possível avaliar se, pela ótica do tomador de decisão, os "ótimos agronômicos" relatados nos estudos de ILP, no tocante à escolha das atividades agropecuárias e às estratégias de rotação, sucessão ou consórcio adotadas, são também opção econômica viável e superior às verificadas em sistemas especializados.

\section{Economia da integração lavoura-pecuária}

Apesar da importância da dimensão econômica para a tomada de decisão, a maioria dos artigos sobre ILP abordam principalmente aspectos agronômicos. Não obstante, trabalhos com foco em aspectos econômicos

Tabela 1. Exemplos de efeitos sinérgicos na integração lavoura-pecuária.

\begin{tabular}{|c|c|c|}
\hline Benefício & Efeito agronômico & Impacto econômico $^{(1)}\left(\mathrm{R} \$\right.$ ha $\left.^{-1}\right)$ \\
\hline & \multicolumn{2}{|c|}{ Pecuária para a lavoura } \\
\hline Eficiência no uso de fertilizantes ${ }^{(2)}$ & Evita a perda de $800 \mathrm{~kg} \mathrm{ha}^{-1}$ de grãos de soja & 600,00 \\
\hline Eficiência no uso de fertilizantes ${ }^{(3)}$ & Ganho de 87 a $1.075 \mathrm{~kg} \mathrm{ha}^{-1}$ de grãos de soja & $65,00-785,00$ \\
\hline Mofo branco ${ }^{(4)}$ & Evita a perda de $394 \mathrm{~kg} \mathrm{ha}^{-1}$ de grãos de feijão & 460,00 \\
\hline \multirow[t]{2}{*}{ Mofo branco $^{(5)}$} & Redução de aplicações de fungicidas & 190,00 a 380,00 \\
\hline & \multicolumn{2}{|c|}{ Lavoura para a pecuária } \\
\hline Efeito residual de adubações ${ }^{(6)}$ & Equivale a $360 \mathrm{~kg} \mathrm{ha}^{-1}$ de $\mathrm{N}$ para o pasto & 680,00 \\
\hline
\end{tabular}


da ILP têm sido disponibilizados (Ambrosi \& Fontaneli, 1994; Yokoyama et al., 1999; Fontaneli et al., 2000; Cezar \& Yokoyama, 2003; Silva Neto \& Retzlaff, 2004; Lazzarotto et al., 2009; Martha Júnior et al., 2009; Pariz et al., 2009; Viana et al., 2010; Wander et al., 2010).

Esses trabalhos refletem condições ecológicas, perfis de sistemas de produção e condições de mercado distintas. Ressalte-se, também, que não tem havido consenso quanto ao instrumental utilizado para a análise econômica. Esse fato limita a comunicação clara e concisa, restringe discussões mais aprofundadas sobre o tema e, em última análise, dificulta a tomada de decisão. A seguir, apresentam-se as bases conceituais para a análise econômica e de risco desses sistemas mistos, que suportam a análise apresentada na seção subsequente.

A discussão quanto ao custo de produção está de acordo com Friedman (1976), Schuh (1976) e Alves (1996, 2008).

\section{Economia de escopo}

Por uma dimensão econômica, os sistemas mistos têm dois benefícios potenciais: a economia de escopo (EE), que resulta em menor custo para uma dada produtividade ou no aumento da produtividade sem incremento proporcional nos custos; e na redução de risco do negócio, pela diversificação das atividades agrícolas na propriedade rural.

A EE reflete a redução no custo unitário, quando mais de dois produtos são produzidos por uma mesma empresa (Langemeier \& Jones, 2000; Gao $\&$ Featherstone, 2008). Formalmente, define-se que $\mathrm{y} \in \mathrm{R}^{\mathrm{M}}{ }_{+}$é um vetor de produtos obtidos a partir de um vetor de insumos $\mathrm{x} \in \mathrm{R}^{\mathrm{N}}{ }_{+}$(Grosskopf et al., 1992). Essa relação tecnológica pode ser resumida pelo conjunto de insumos requeridos:

$$
\mathrm{L}(\mathrm{y})=\{\mathrm{x}: \mathrm{x} \text { produz } \mathrm{y}\} .
$$

Neste caso, a função custo é definida como $\mathrm{c}(\mathrm{y}, \mathrm{w})=\min \left\{\mathrm{w}^{*} \mathrm{x}: \mathrm{x} \in \mathrm{L}(\mathrm{y})\right\}$, em que $\mathrm{w} \in \mathrm{R}^{\mathrm{N}}+$ é o vetor de preços dos insumos.

Para o caso de dois produtos $-\mathrm{y}=\left(\mathrm{y}^{1}, \mathrm{y}^{2}\right)-$, a economia de escopo irá existir se: $\mathrm{c}\left(\mathrm{y}^{1}, 0: \mathrm{w}\right)+\mathrm{c}\left(0, \mathrm{y}^{2}: \mathrm{w}\right)>\mathrm{c}\left(\mathrm{y}^{1}, \mathrm{y}^{2}: \mathrm{w}\right)$. A deseconomia de escopo ocorre se a inegualdade for invertida. De maneira simplificada, tem-se $\mathrm{EE}=[\Sigma(\mathrm{Yi})-\mathrm{C}(\mathrm{Y})] / \mathrm{C}(\mathrm{Y})]$, em que: $\mathrm{C}(\mathrm{Yi})$ é o custo de se produzir somente $\mathrm{Yi}$ em empreendimentos separados; e C(Y) é o custo de se produzirem todos os produtos em uma única empresa multiproduto.

Se o resultado da equação para EE for positivo, há economia de escopo. Isso implica que a empresa multiproduto - diversificada - é mais eficiente em termos de custos. Como extensão, a EE ocorre quando a produção de múltiplos produtos na fazenda, de modo integrado, aumenta a produtividade. Em outras palavras, a EE é verificada quando há complementaridade entre as atividades na fazenda, o que torna possível que uma dada atividade aumente a produtividade marginal de outra (Chavas \& Kim, 2007).

\section{Diversificação e redução do risco}

Os produtores rurais desenvolvem suas atividades em um ambiente dinâmico e incerto, em que variáveis econômicas e físicas encontram-se em constante mudança (Dent et al., 1986). Conforme a percepção do risco do negócio, os produtores rurais podem optar por atividades de menor risco, mesmo que isso implique comprometimento da receita média da fazenda. Esse comportamento é condizente com o fato de que a maioria dos produtores rurais é avessa ao risco (Barry et al., 2000; Chavas, 2008; Moss, 2010).

Cabe lembrar que ser avesso ao risco não significa que os indivíduos não correm risco. Significa que os indivíduos precisam ser compensados para correr riscos, e que essa compensação deve aumentar conforme o risco, ou o nível de aversão ao risco aumenta. Note-se que o nível de aversão ao risco dos indivíduos pode variar com o tempo, em razão da riqueza, experiências anteriores etc. (Barry et al., 2000).

A ILP pode atuar na redução de risco do negócio, pela diversificação das atividades agropecuárias na propriedade rural. Além desse efeito de diversificação, a rotação de culturas na propriedade rural pode contribuir positivamente para a redução de risco, pela menor variabilidade da produtividade entre anos e pelo aumento na produtividade ou redução dos custos unitários de produção (Helmers et al., 2001). Contudo, em uma situação de falta de crédito, em que há necessidade de retorno mais rápido do investimento (Antle \& Diagana, 2003), uma maior percepção de risco pode ser agregada ao agente econômico, o que desestimularia investimentos nessas tecnologias.

Apenas com correlações perfeitamente positivas entre os retornos dos produtos, não haveria efeito da diversificação sobre a redução do risco. Com elevada correlação positiva, o efeito de redução do risco 
do portfólio é marginal. Efeitos mais pronunciados demandariam a associação de atividades com correlação fraca, próxima de zero, ou, para efeitos mais expressivos, a inclusão de atividades com correlação negativa no portfólio.

Barry et al. (2000) apontaram que a diversificação com culturas de grãos oferece oportunidades limitadas para a redução de risco. Isso ocorre porque, numa dada localidade, as culturas de grãos experimentam padrões climáticos semelhantes, utilizam recursos semelhantes e estão sujeitas a fatores de mercado similares, o que determina correlações positivas entre as atividades, geralmente de elevada magnitude. Entre soja e pecuária de engorda, Barry et al. (2000) apontaram correlação negativa.

Cabe lembrar que é possível a redução de risco em sistemas especializados por meio de outros mecanismos. A diversificação geográfica pode ser uma alternativa. Há necessidade de que a eventual redução nos riscos, pela distribuição da produção em diferentes locais, seja confrontada com o impacto sobre o resultado econômico.

Outro mecanismo seria o de formação de portfólios que envolvam outros ativos, que não atividades agropecuárias, como aplicações financeiras, por exemplo. O próprio progresso tecnológico, nas últimas décadas, tem contribuído para a redução da exposição ao risco. Traxler et al. (1995) observaram que, em razão do progressivo sucesso do melhoramento genético, tem havido ganhos em estabilidade produtiva, aumento na produtividade média e, em alguns casos, ambos os efeitos. Esse enfraquecimento dos efeitos do risco nas estratégias de diversificação, de acordo com Chavas (2008), pode indicar que o progresso tecnológico está sendo importante fator para a maior especialização na agricultura.

\section{Análise ex ante da integração lavoura-pecuária}

Não se dispõe de um número suficientemente grande de experimentos que tenham quantificado, simultaneamente, as interações entre os componentes pasto e lavoura em termos agronômicos e econômicos. Este motivo e o fato de, geralmente, não haver nesses experimentos acompanhamento anual da produção agropecuária, no sistema misto e nos respectivos sistemas especializados, restringem a possibilidade de se estimarem com precisão as interações, as funções custo para fazendas multiprodutos e o custo de oportunidade do uso da terra.

O modelo de avaliação proposto neste trabalho baseiase no custo de produção. Partindo-se de duas atividades, A e B, a pergunta relevante é: qual o desempenho econômico que a atividade $\mathrm{B}$ tem de ter, para competir com a atividade $\mathrm{A}$ ? No custo total da atividade B, devese adicionar a renda líquida da atividade A; satisfeita esta condição, ou seja, se a renda líquida de B for maior do que esse custo de oportunidade, o produtor migrará da atividade A para a B.

Desse modo, a ILP será preferível aos empreendimentos especializados - pecuária e soja, por exemplo - se a renda líquida gerada nos sistemas mistos, depois de as interações (positivas e negativas) entre pasto e lavoura terem sido computadas, for superior ao custo de oportunidade em sistemas especializados. Portanto, $\mathrm{RB}_{\text {ILP }}-\left(\mathrm{CT}_{\mathrm{ILP}}+\mathrm{RL}_{\mathrm{Esp}}\right)>0$, em que: $\mathrm{RB}_{\text {ILP }}$ é a receita bruta da ILP; $\mathrm{CT}_{\text {ILP }}$, o custo total da ILP; e $\mathrm{RL}_{\mathrm{Esp}}$, a receita líquida do sistema especializado. Ressalte-se que, para o empreendedor, que por definição nada possui, os custos totais equivalem ao total de desembolsos que remuneram as compras e serviços de terra, trabalho e capital, que pertencem ao capitalista.

Esse raciocínio é igualmente pertinente para se investigar a competição pelo uso da terra entre atividades agropecuárias.

Para prosseguir na análise, é necessário computar as taxas de retorno do empreendedor, que medem o retorno do empreendimento por unidade $(\mathrm{R} \$$ ) de custo total das atividades em análise. Ressalte-se que o produtor pode ter outras razões para a tomada de decisão além de uma análise econômica rigorosa.

A análise ex ante a seguir aplica esses conceitos. Os pressupostos econômicos são os expostos na seção: economia da integração lavoura-pecuária.

\section{Caracterização dos sistemas}

A área ocupada pelas atividades agropecuárias, nos cenários testados (pecuária extensiva de corte, soja, ILP com pecuária de corte), é de 1.500 ha. No sistema ILP, $50 \%$ da área é tomada pela pecuária e o restante pela soja; há rotação no uso da terra a cada dois anos.

As taxas de lotação e o desempenho animal para a pecuária, na ILP e no sistema especializado, são consistentes com os valores indicados por Martha Júnior et al. (2009, 2010a). Considerou-se que a produtividade média anual da pecuária, na ILP, foi de $537 \mathrm{~kg} \mathrm{ha}^{-1}$ de peso vivo (PV), e a da pecuária, no 
sistema especializado, de $211 \mathrm{~kg} \mathrm{ha}^{-1}$ de PV por ano. A produtividade média de soja, de $2.880 \mathrm{~kg} \mathrm{ha}^{-1}$, é a estimativa apresentada pela Conab para a safra de 2009/2010, em Goiás. Os demais coeficientes técnicos, como as quantidades de insumos utilizadas nos sistemas de produção, foram obtidos junto ao banco de dados e ao corpo técnico da Embrapa.

Os preços dos insumos e produtos referem-se aos valores de mercado (Goiás) do último trimestre de 2010. O preço da arroba do boi gordo, para fim de análise, foi de $\mathrm{R} \$ 92,25$. O garrote, para a reposição, custou R\$965,00. A soja apresentou preço de $\mathrm{R} \$ 45,00$ por saca. Esses valores variam, quando análises de sensibilidade para preços são aplicadas.

\section{Resultado econômico}

A pecuária, no sistema especializado, apresentou renda líquida negativa ( $\mathrm{R} \$-30,03 \mathrm{ha}^{-1}$ ) (Tabela 2). Os valores de renda líquida para a soja ( $\mathrm{R} \$ 774,39 \mathrm{ha}^{-1}$ ) e para a integração lavoura-pecuária (R\$ 718,43 ha-1) foram semelhantes. Entretanto, as taxas de retorno do empreendedor diferiram bastante entre os sistemas. Para a pecuária, no sistema especializado, o valor foi negativo $(-1,55 \%)$; para a ILP, a taxa de retorno foi de $26,7 \%$ e, para a soja, de $55,9 \%$. Isso se deve ao maior dispêndio total na ILP (R \$ 2.691,31 ha $\left.{ }^{-1}\right)$ em comparação aos sistemas especializados ( $\mathrm{R} \$ 1.385,61 \mathrm{ha}^{-1}$, para a soja e R\$1.931,73 ha-1, para a pecuária extensiva), principalmente em razão da elevada demanda de capital para a compra de animais de reposição. Notese que a pecuária, no sistema especializado, mesmo refletindo um sistema com uso de insumos de baixo a moderado, foi mais intensiva em capital do que a cultura da soja, também em razão dos gastos elevados com animais para a reposição.

Há evidência de economia de escopo nos resultados apresentados na Tabela 2, em especial para a pecuária. $O$ custo da soja manteve-se praticamente inalterado na ILP (R\$ 29,59 por saca), em comparação ao sistema especializado ( $\mathrm{R} \$ 28,87$ por saca). Contudo, verificouse efeito expressivo no caso da arroba (vendida) do boi gordo, tendo-se como base um garrote com $315 \mathrm{~kg}$ de peso vivo. O custo, no sistema especializado de pecuária, foi de R \$ 93,05 por arroba e, na pecuária da ILP passou para $\mathrm{R} \$ 77,88$ por arroba.

Pela ótica econômica, ter renda líquida positiva na atividade é condição necessária, porém, não suficiente para escolher uma alternativa de uso da terra vis-à-vis outra atividade. É preciso considerar o custo de oportunidade.

A aplicação da equação para $\mathrm{RB}_{\mathrm{ILP}}$, na comparação entre pecuária e ILP, indica que a renda líquida da ILP cobre o custo de oportunidade do sistema especializado, em todos os cenários de produtividade da soja testados (Tabela 3). Tomando-se uma produtividade de 48 sacas ha $^{-1}$ de soja na ILP e uma produtividade na fase de pecuária da ILP de $537 \mathrm{~kg} \mathrm{ha}^{-1}$ de PV (correspondente as 3 cabeças ha- ${ }^{-1}$ e ganho de peso de $179 \mathrm{~kg}$ por cabeça ao

Tabela 2. Descrição e resultado econômico projetado dos sistemas avaliados.

\begin{tabular}{|c|c|c|c|c|c|}
\hline \multirow[t]{2}{*}{ Parâmetro } & \multirow[t]{2}{*}{ Pecuária extensiva } & \multirow[t]{2}{*}{ Soja } & \multicolumn{3}{|c|}{ Integração lavoura pecuária } \\
\hline & & & Total & Pecuária & Soja \\
\hline & \multicolumn{5}{|c|}{ Dados de entrada } \\
\hline Área com pasto (ha) & 1.500 & 0 & 750 & - & - \\
\hline Área com soja (ha) & 0 & 1.500 & 750 & - & - \\
\hline Produtividade soja $\left(\mathrm{kg} \mathrm{ha}^{-1}\right)$ & - & 2.880 & 2.880 & - & - \\
\hline Produtividade pecuária $\left(\mathrm{kg} \mathrm{ha}^{-1} \mathrm{de} \mathrm{PV}\right)$ & 211 & - & 537 & - & - \\
\hline Preço soja (R\$ por saca) & - & 45,00 & 45,00 & - & - \\
\hline \multirow[t]{2}{*}{ Preço pecuária ( $\mathrm{R} \$$ por arroba) } & 92,25 & - & 92,25 & - & - \\
\hline & \multicolumn{5}{|c|}{ Resultados } \\
\hline Custeio $\left(\mathrm{R} \$ \mathrm{ha}^{-1}\right)$ & $1.406,00$ & 940,62 & $2.143,94$ & $3.348,68$ & 939,20 \\
\hline Depreciações $\left(\mathrm{R} \$\right.$ ha $\left.^{-1}\right)$ & 81,23 & 45,40 & 72,67 & 72,67 & 72,67 \\
\hline \multicolumn{6}{|l|}{ Custo operacional } \\
\hline Custeio ( $\mathrm{R} \$$ ha $\left.^{-1}\right)$ & 77,33 & 51,73 & 117,92 & 184,18 & 51,66 \\
\hline Bens de capital & 367,18 & 347,85 & 356,78 & 356,78 & 356,78 \\
\hline Dispêndio (D) $\left(\mathrm{R} \$\right.$ ha $\left.^{-1}\right)$ & $1.931,74$ & $1.385,61$ & $2.691,31$ & $3.962,31$ & $1.420,31$ \\
\hline Receita bruta $(\mathrm{RB})\left(\mathrm{R} \$\right.$ ha $\left.^{-1}\right)$ & $1.901,71$ & $2.160,00$ & $3.409,73$ & $4.659,47$ & $2.160,00$ \\
\hline $\mathrm{RB}-\mathrm{D}\left(\mathrm{R} \$ \mathrm{ha}^{-1}\right)$ & $-30,03$ & 774,39 & 718,43 & 697,16 & 739,69 \\
\hline Retorno $[(\mathrm{RB} \quad \mathrm{D}) / \mathrm{D}](\%)$ & $-1,55$ & 55,89 & 26,69 & 18,60 & 52,08 \\
\hline
\end{tabular}


ano), a renda líquida no sistema misto supera aquela na pecuária em $\mathrm{R} \$ 748,46 \mathrm{ha}^{-1}$. O diferencial de taxa de retorno para a ILP foi superior a $28 \%$.

A aplicação da equação para $\mathrm{RB}_{\mathrm{ILP}}$, na comparação entre soja e ILP, indica que a renda líquida da ILP cobre o custo de oportunidade do sistema especializado, quando o preço da soja é inferior a $\mathrm{R} \$$ 30,00 por saca e a produtividade da pecuária na ILP é superior a $358 \mathrm{~kg} \mathrm{ha}^{-1}$ de PV (taxa de lotação na ILP superior a 2,0 cabeças ha ${ }^{-1}$ e ganho de peso de $179 \mathrm{~kg}$ por cabeça). Cada incremento de $\mathrm{R} \$ 1,00$ no preço da saca de soja, na faixa de $R \$ 35,00$ a 50,00 por saca, demanda aumento adicional de aproximadamente $18 \mathrm{~kg}$ de peso vivo na produtividade da pecuária de engorda (0,1 cabeça por hectare, $179 \mathrm{~kg}$ por cabeça), para cobrir o custo de oportunidade da soja. Quando a soja atinge $\mathrm{R} \$ 55,00$ por saca, a ILP não cobre o custo de oportunidade da oleaginosa em nenhum cenário de produtividade testado (Tabela 4 ).

$\mathrm{Na}$ análise de sensibilidade para produtividade na ILP, por meio de variação nos coeficientes técnicos da soja e da pecuária, observa-se que para a ILP cobrir o custo de oportunidade da soja, considerando-se preços de $\mathrm{R} \$ 45,00$ por saca e $\mathrm{R} \$ 92,25$ por arroba de boi gordo, a produtividade de soja na ILP tem de ser semelhante àquela do sistema especializado (neste caso, 48 sacas ha $^{-1}$ ), e a produtividade da pecuária tem que ser elevada e da ordem de 21,5 arrobas ha-1 $(3,5$ cabeças por hectare e ganho de peso de $179 \mathrm{~kg}$ por cabeça) (Tabela 5).

Ressalte-se que, embora haja ocasiões em que a ILP cobre o custo de oportunidade da soja (Tabelas 4 e 5), a tecnologia não apresentou taxas de retorno (do empreendedor) competitivas com a soja, nos cenários testados. Tomando-se os meses de junho a setembro como os norteadores para as decisões de plantio (indicadores Cepea/BMF-Bovespa para boi gordo e soja, disponíveis em www.cepea.esalq.usp.br), os preços relativos da arroba do boi gordo para a saca de soja oscilaram de 1,42 a 2,15, com média de 1,84 arroba de boi gordo por saca de soja. Para essa faixa de preços relativos, a decisão não se altera sensivelmente, isto é, a taxa de retorno da ILP não cobre a verificada para a soja.

Tabela 3. Renda líquida da integração lavoura-pecuária $\left(\mathrm{R} \$ \mathrm{ha}^{-1}\right)$, considerando-se diferentes produtividades da lavoura de soja e da pecuária, no sistema de integração lavoura pecuária, depois de se computar o custo de oportunidade da pecuária em sistema especializado ${ }^{(1)}$.

\begin{tabular}{|c|c|c|c|c|c|c|c|}
\hline \multirow{2}{*}{$\begin{array}{l}\text { Produtividade da pecuária } \\
\left(\mathrm{kg} \mathrm{ha}^{-1} \text { de } \mathrm{PV}\right)\end{array}$} & \multicolumn{7}{|c|}{ Produtividade da soja (sacas ha-1) } \\
\hline & 43 & 45 & 47 & 48 & 49 & 51 & 53 \\
\hline 268,5 & 318,28 & 361,54 & 404,80 & 426,43 & 448,06 & 491,32 & 534,58 \\
\hline 358,0 & 425,96 & 469,22 & 512,48 & 534,11 & 555,74 & 599,00 & 642,26 \\
\hline 447,5 & 532,63 & 575,89 & 619,15 & 640,78 & 662,41 & 705,67 & 748,93 \\
\hline 537,0 & 640,31 & 683,57 & 726,83 & 748,46 & 770,09 & 813,35 & 856,60 \\
\hline 626,5 & 747,99 & 791,24 & 834,50 & 856,13 & 877,76 & 921,02 & 964,28 \\
\hline 716,0 & 854,65 & 897,91 & 941,17 & 962,80 & 984,43 & $1.027,69$ & $1.070,95$ \\
\hline
\end{tabular}

(1)Produtividade da pecuária no sistema especializado igual a $211 \mathrm{~kg} \mathrm{ha}^{-1} \mathrm{de} \mathrm{PV}$.

Tabela 4. Renda líquida da integração lavoura-pecuária $\left(\mathrm{R} \$ \mathrm{ha}^{-1}\right)$, considerando-se diferentes produtividades da pecuária no sistema de integração lavoura-pecuária e preços de soja, depois de se computar o custo de oportunidade da soja em sistema especializado $^{(1)}$.

\begin{tabular}{|c|c|c|c|c|c|c|c|}
\hline \multirow{2}{*}{$\begin{array}{l}\text { Produtividade da pecuária } \\
\left(\mathrm{kg} \mathrm{ha}^{-1} \text { de } \mathrm{PV}\right)\end{array}$} & \multicolumn{7}{|c|}{ Preço da soja (R\$ por saca) } \\
\hline & 25 & 30 & 35 & 40 & 45 & 50 & 55 \\
\hline 268,5 & 107,07 & $-14,19$ & $-135,46$ & $-256,72$ & $-377,99$ & $-499,26$ & $-620,52$ \\
\hline 358,0 & 214,75 & 93,49 & $-27,78$ & $-149,05$ & $-270,31$ & $-391,58$ & $-512,84$ \\
\hline 447,5 & 321,42 & 200,15 & 78,89 & $-42,38$ & $-163,64$ & $-284,91$ & $-406,18$ \\
\hline 537,0 & 429,10 & 307,83 & 186,57 & 65,30 & $-55,97$ & $-177,23$ & $-298,50$ \\
\hline 626,5 & 536,78 & 415,51 & 294,24 & 172,98 & 51,71 & $-69,55$ & $-190,82$ \\
\hline 716,0 & 643,45 & 522,18 & 400,91 & 279,65 & 158,38 & 37,12 & $-84,15$ \\
\hline
\end{tabular}

${ }^{(1)}$ Produtividade da soja em sistema especializado igual a 48 sacas ha $^{-1}$. 
A Tabela 6 apresenta informações para se saber qual o preço da soja (todo o resto constante) necessário para que os sistemas de soja especializada e ILP apresentem taxas de retorno do empreendedor semelhantes. Considera-se o cenário atual de preços e faz-se uma análise de sensibilidade para os preços dos fertilizantes, aplicados à soja (não há adubação do pasto) de $30 \mathrm{a}$ $41 \%$ das despesas totais.

De modo geral, os preços em que a ILP seria competitiva com o sistema especializado de lavoura estão próximos a $\mathrm{R} \$ 35,00$ por saca. As taxas de retorno são semelhantes entre os dois sistemas, ao redor de 16,5\%. A renda líquida da ILP (cerca de $\mathrm{R} \$ 450,00)$, em média, é $80 \%$ superior à observada para a lavoura especializada de soja (cerca de R $\$ 240,00$ ). É inevitável pontuar que trabalhos futuros precisam explorar outras alternativas de ILP que contemplem, além de outras possibilidades agronômicas, diferentes termos de troca e preços relativos de produtos. Além disso, é preciso avançar na quantificação do tamanho do risco, em termos econômicos, para tornar os sistemas mistos competitivos.

\section{Considerações finais}

A tomada de decisão em prol de sistemas especializados ou mistos deve variar em função dos preços relativos de produtos e insumos. Nos cenários testados, a integração lavoura-pecuária (ILP) compete com sistemas especializados de pecuária, mas não apresenta taxas de retorno competitivas em comparação a sistemas especializados de soja.

Retornos econômicos mais favoráveis nos sistemas mistos dependem da elevada produtividade das lavouras e da pecuária. A produtividade elevada aumenta a demanda por insumos modernos e por bens de capital.

Tabela 5. Renda líquida na integração lavoura-pecuária $\left(\mathrm{R} \$ \mathrm{ha}^{-1}\right)$, considerando-se diferentes produtividades da lavoura de soja e da pecuária no sistema de integração lavoura-pecuária, depois de se computar o custo de oportunidade da soja em sistema especializado ${ }^{(1)}$.

\begin{tabular}{|c|c|c|c|c|c|c|c|}
\hline \multirow{2}{*}{$\begin{array}{l}\text { Produtividade da pecuária } \\
\left(\mathrm{kg} \mathrm{ha}^{-1} \text { de } \mathrm{PV}\right)\end{array}$} & \multicolumn{7}{|c|}{ Produtividade da soja $\left(\right.$ sacas ha ${ }^{-1}$ ) } \\
\hline & 43 & 45 & 47 & 48 & 49 & 51 & 53 \\
\hline 268,5 & 486,14 & 442,88 & 399,62 & 377,99 & 356,36 & 313,10 & 269,84 \\
\hline 358,0 & 378,46 & 335,20 & 291,94 & 270,31 & 248,68 & 205,42 & 162,16 \\
\hline 447,5 & 271,79 & 228,53 & 185,27 & 163,64 & 142,01 & 98,75 & 55,49 \\
\hline 537,0 & 164,11 & 120,85 & 77,59 & 55,97 & 34,34 & 8,92 & 52,18 \\
\hline 626,5 & 56,44 & 13,18 & 30,08 & 51,71 & 73,34 & 116,60 & 159,86 \\
\hline 716,0 & 50,23 & 93,49 & 136,75 & 158,38 & 180,01 & 223,27 & 266,53 \\
\hline
\end{tabular}

(1) Produtividade da soja em sistema especializado igual a 48 sacas ha-1. $^{-1}$

Tabela 6. Desempenho econômico da soja e da integração lavoura-pecuária (ILP), para taxas de retorno do empreendedor semelhantes, considerando-se alterações nos preços de fertilizantes e nas relações entre preço da arroba do boi gordo e da saca de soja (arroba de boi por saca).

\begin{tabular}{|c|c|c|c|c|c|}
\hline Atividade & Renda bruta & $\begin{array}{l}\text { Despesa total } \\
---\left(\mathrm{R} \$ \text { ha }^{-1}\right)\end{array}$ & Renda líquida & $\begin{array}{c}\text { Taxa de retorno do empreendedor } \\
(\%)\end{array}$ & $\mathrm{R} \$$ por saca de soja \\
\hline & \multicolumn{5}{|c|}{ Preços correntes de fertilizantes ( $30 \%$ das despesas totais); arroba por saca $=2,76$} \\
\hline Soja & $1.606,88$ & $1.379,77$ & 227,10 & 16,46 & - \\
\hline \multirow[t]{2}{*}{ ILP } & $2.688,39$ & $3.133,17$ & 444,78 & 16,54 & 33,48 \\
\hline & \multicolumn{5}{|c|}{ Preços correntes de fertilizantes $+10 \%$ (33\% das despesas totais); arroba por saca $=2,68$} \\
\hline Soja & $1.654,97$ & $1.419,69$ & 235,28 & 16,57 & \\
\hline \multirow[t]{2}{*}{ ILP } & $3.157,22$ & $2.708,35$ & 448,87 & 16,57 & 34,48 \\
\hline & \multicolumn{5}{|c|}{ Preços correntes de fertilizantes $+25 \%$ ( $36 \%$ das despesas totais); arroba por saca $=2,57$} \\
\hline Soja & $1.724,71$ & $1.479,53$ & 245,18 & 16,57 & \\
\hline \multirow[t]{2}{*}{ ILP } & $3.192,09$ & $2.738,27$ & 453,82 & 16,57 & 35,93 \\
\hline & \multicolumn{5}{|c|}{ Preços correntes de fertilizantes $+50 \%$ ( $41 \%$ das despesas totais); arroba por saca $=2,41$} \\
\hline Soja & $1.840,91$ & $1.579,26$ & 261,65 & 16,57 & - \\
\hline ILP & $3.250,19$ & $2.788,14$ & 462,05 & 16,57 & 38,35 \\
\hline
\end{tabular}


Assegurar amplo acesso a esses fatores de produção é estratégia necessária para garantir atratividade à ILP.

A alta demanda por capital, particularmente para a aquisição de animais para a manutenção de elevadas (e necessárias) taxas de lotação na ILP - que podem representar até $50 \%$ dos custos totais de produção explica as menores taxas de retorno da ILP e é vista como uma das principais restrições para a ampla adoção desses sistemas mistos.

Sem incentivos, é provável que, em regiões de lavouras produtivas, seja lenta a adoção do sistema de integração lavoura-pecuária, a qual depende de preços relativos mais favoráveis e da disponibilidade de linhas de crédito que melhor se adequem às necessidades do produtor, em termos de volume de recursos e prazos de pagamento.

A integração lavoura-pecuária pode ter efeito favorável sobre a redução do risco de produção e de preço, pela diversificação de atividades. Entretanto, a alta demanda por capital desses sistemas aumenta o risco financeiro do empreendimento.

Há o risco de se perderem ganhos potenciais com economia de escopo, em razão das ineficiências que surgem da aplicação inadequada da tecnologia ILP. Se isso acontecer, o sistema de integração lavoura-pecuária, que poderia contribuir positivamente com objetivos privados e públicos, é preterido em prol de sistemas especializados de menor grau de complexidade.

É preciso delinear arranjos experimentais que permitam estimar, com precisão, interações entre os componentes lavoura e pecuária e a função custo desses sistemas mistos vis-à-vis aquela de sistemas especializados. Esses estudos devem viabilizar análises econômicas e de risco mais robustas, que contribuam de modo efetivo para a tomada de decisão de agentes públicos e privados.

Merece investigação mais detalhada a competitividade da integração lavoura-pecuária $v i s-\grave{a}$-vis tecnologias em uso, em resposta a variações nas taxas de juros e preços relativos no mercado interno, à volatilidade dos preços externos e ao comportamento do risco em sistemas mistos e especializados. Analisar a recuperação de pastagens por meio da ILP vis-à-vis outras alternativas, como a adubação de pastagens, também é tema relevante.

Em razão das dimensões continentais do país, é importante que se realizem essas análises nas diferentes regiões produtoras.

\section{Agradecimentos}

À Financiadora de Estudos e Projetos, ao Conselho Nacional de Desenvolvimento Científico e Tecnológico, à Fundação de Amparo à Pesquisa do Distrito Federal e à Embrapa, pelo apoio financeiro.

\section{Referências}

ALVES, E. A função custo. Brasília: Embrapa-SPI, 1996. 106p.

ALVES, E. Teoria da produção: métodos não paramétricos. Brasília: Embrapa Informação Tecnológica, 2008. 87p.

AMBROSI, I.; FONTANELI, R.S. Análise de risco de quatro sistemas alternativos de produção de integração lavoura/pecuária. Teoria e Evidência Econômica, v.2, p.119-135, 1994.

ANTLE, J.M.; DIAGANA, B. Creating incentives for the adoption of sustainable agricultural practices in developing countries: the role of soil carbon sequestration. American Journal of Agricultural Economics, v.85, p.1178-1184, 2003.

AYARZA, M.A.; VILELA, L.; PIZARRO, E.A.; COSTA, P.H. da. Agropastoral systems based on legumes: an alternative for sustainable agriculture in the Brazilian Cerrados. In: THOMAS, R.E.; AYARZA, M.A. (Ed.). Sustainable land management for the oxisols of the Latin American savannas. Cali: CIAT, 1999. p.22-36. (Publicación CIAT, 312).

BARRY, P.J.; ELLINGER, P.N.; HOPKIN, J.A.; BAKER, C.B. Financial management in agriculture. $6^{\text {th }}$ ed. Danville: Interstate, 2000. 678p.

CEZAR, I.M.; YOKOYAMA, L.P. Avaliação bioeconômica de recuperação de pastagens pelo sistema Barreirão: estudo de casos. In: KLUTHCOUSKI, J.; STONE, L.F.; AIDAR, H. (Ed.). Integração lavoura-pecuária. Santo Antônio de Goiás: Embrapa Arroz e Feijão, 2003. p.363-383.

CHAVAS, J.-P. On the economics of agricultural production. The Australian Journal of Agricultural and Resource Economics, v.52, p.365-380, 2008.

CHAVAS, J.-P.; KIM, K. Measurement and sources of economies of scope: a primal approach. Journal of Institucional and Theoretical Economics, v.163, p.411-427, 2007.

COSTA, J.L.S. Influência da braquiária no manejo de doenças do feijoeiro com origem no solo. In: KLUTHCOUSKI, J.; STONE, J.L.; AIDAR, H. (Ed.) Integração lavoura-pecuária. Santo Antônio de Goiás: Embrapa Arroz e Feijão, 2003. p.523-538.

DEDECEK, R.A.; RESCK, D.V.S.; FREITAS JUNIOR, E. de. Perdas de solo, água e nutrientes por erosão em Latossolo Vermelho-Escuro dos dos cerrados em diferentes cultivos sob chuva natural. Revista Brasileira de Ciência do Solo, v.10, p.265-272, 1986 .

DENT, J.B.; HARRISON, S.R.; WOODFORD, K.B. Farm planning with linear programming: concept and practice. Sydney: Butterworth-Heinemann, 1986. 209p.

FONTANELI, R.S.; AMBROSI, I.; SANTOS, H.P. dos; IGNACZAK, J.C.; ZOLDAN, S.M. Análise econômica de 
sistemas de produção de grãos com pastagens anuais de inverno em sistema de plantio direto. Pesquisa Agropecuária Brasileira, v.35, p.2129-2137, 2000.

FRIEDMAN, M. Price theory. Chicago: Aldine Publishing Company, 1976. 357p.

GAO, Z.; FEATHERSTONE, A.M. Estimating economies of scope using profit function: a dual approach for the normalized quadratic profit function. Economic Letters, v.100, p.418-421, 2008.

GARAGORRY, L.F.; ALVES, E.; SOUZA, G. da S. e. Tipos de especialização na agricultura brasileira. Revista Brasileira de Economia, v.57, p.337-368, 2003.

GOUVELlO, C. de. Estudo de baixo carbono para o Brasil. Brasília: Banco Mundial, 2010. 278p.

GROSSKOPF, S.; HAYES, K.; YAISAWARNG, S. Measuring economies of diversification: a frontier approach. Journal of Business and Economic Statistics, v.10, p.453-459, 1992.

HELMERS, G.A.; YAMOAH, C.F.; VARVEL, G.E. Separating the impacts of crop diversification and rotations on risk. Agronomy Journal, v.93, p.1337-1340, 2001.

HERRERO, M.; THORNTON, P.K.; NOTENBAERT, A.M.; WOOD, S.; MSANGI, S.; FREEMAN, H.A.; BOSSIO, D.; DIXON, J.; PETERS, M.; STEEG, J. van de; LYNAM, J.; PARTHASARATHY RAO, P.; MACMILLAN, S.; GERARD, B.; MCDERMOTT, J.; SERÉ, C.; ROSEGRANT, M. Smart investments in sustainable food production: revisiting mixed crop-livestock systems. Science, v.327, p.822-825, 2010.

KLUTHCOUSKI, J.; STONE, L.F.; AIDAR, H. Integração lavoura-pecuária. Santo Antônio de Goiás: Embrapa Arroz e Feijão, 2003. 570p.

LANGEMEIER, M.R.; JONES, R.D. Measuring the impact of farm size and specialization on financial performance. Journal of the ASFMRA, p.90-96, 2000.

LAZZAROTTO, J.J.; SANTOS, M.L. dos; LIMA, J.E. de; MORAES, A. Volatilidade dos retornos econômicos associados à integração lavoura-pecuária no Estado do Paraná. Revista de Economia e Agronegócio, v.7, p.259-283, 2009.

MARTHA JÚNIOR, G.B.; ALVES, E.; MUELLER, C.C.; VILELA, L. Análise econômica e de risco da pecuária extensiva no Cerrado. In: CONGRESSO DA SOCIEDADE BRASILEIRA DE ECONOMIA, ADMINISTRAÇÃO E SOCIOLOGIA RURAL, 48., 2010, Campo Grande. Anais. Campo Grande: SOBER, 2010a. 18p.

MARTHA JÚNIOR, G.B.; MUELLER, C.C.; ALVES, E.R. de A.; VILELA, L. Análise ex-ante do desempenho econômicofinanceiro de alternativas de integração lavoura-pecuária no Triângulo Mineiro e no sudoeste Goiano. Planaltina: Embrapa Cerrados, 2009. 26p. (Embrapa Cerrados. Boletim de pesquisa e desenvolvimento, 262).

MARTHA JÚNIOR, G.B.; VILELA, L.; SOUSA, D.M.G. de. Adubação nitrogenada. In: MARTHA JÚNIOR, G.B.; VILELA, L.; SOUSA, D.M.G. (Ed.). Cerrado: uso eficiente de corretivos e fertilizantes em cerrados. Planaltina: Embrapa Cerrados, 2007. p.117-144.

MARTHA, JÚNIOR, G.B.; VILELA, L.; SOUSA, D.M.G. de. Integração lavoura-pecuária. In: SIMPÓSIO SOBRE BOAS
PRÁTICAS PARA USO EFICIENTE DE FERTILIZANTES, 2009, Piracicaba. Boas práticas para uso eficiente de fertilizantes: anais. Piracicaba: IPNI, 2010b. v.3, p.1111-1131.

MAUGHAN, M.W.; FLORES, J.P.C.; ANGHINONI, I.; BOLLERO, G.; FERNANDÉZ, F.G.; TRACY, B.F. Soil quality and corn yield under crop-livestock integration in Illinois. Agronomy Journal, v.101, p.1503-1510, 2009.

MIRANDA, J.C.C. de; VILELA, L.; MIRANDA, L.N. de. Dinâmica e contribuição da micorriza arbuscular em sistemas de produção com rotação de culturas. Pesquisa Agropecuária Brasileira, v.40, p.1005-1014, 2005.

MOSS, C.B. Risk, uncertainty and the agricultural firm. New Jersey: World Scientific, 2010. 292p.

PARIZ, C.M.; ANDREOTTI, M.; TARSITANO, M.A.A.; BERGAMASCHINE, A.F.; BUZETTI, S.; CHIODEROLI, C.A. Desempenhos técnicos e conômicos da consorciação de milho com forrageiras dos gêneros Panicum e Brachiaria em sistema de integração lavoura-pecuária. Pesquisa Agropecuária Tropical, v.39, p.360-370, 2009.

PORTES, T. de A.; CARVALHO, S.I.C. de; OLIVEIRA, I.P. de; KLUTHCOUSKI, J. Análise do crescimento de uma cultivar de braquiária em cultivo solteiro e consorciado com cereais. Pesquisa Agropecuária Brasileira, v.35, p.1349-1358, 2000.

RICARDO, T.R.; WANDER, A.E.; LOBO JUNIOR, M.; PICANÇO FILHO, A.F. Custos associados ao mofo branco (Sclerotinia esclerotiorum) em feijoeiro comum de $3^{\text {a }}$ safra em Goiás. In: CONGRESSO DA SOCIEDADE BRASILEIRA DE ECONOMIA, ADMINISTRAÇÃO E SOCIOLOGIA RURAL, 47., 2009, Porto Alegre. Anais. Porto Alegre: SOBER, 2010. 8p.

SCHUH, G.E. Considerações teóricas sobre custos de produção na agricultura. Agricultura em São Paulo, v.23, p.97-119, 1976.

SILVA NETO, B.; RETZLAFF, E. Otimização sob incerteza de sistemas de produção: integração lavoura-pecuária com ênfase em bovinocultura de leite. Ciência Rural, v.34, p.1207-1212, 2004.

SOUSA, D.M.G. de; MARTHA JÚNIOR, G.B.; VILELA, L. Adubação fosfatada. In: MARTHA JÚNIOR, G.B.; VILELA, L.; SOUSA, D.M.G. (Ed.). Cerrado: uso eficiente de corretivos e fertilizantes em cerrados. Planaltina: Embrapa Cerrados, 2007. p.145-177.

SOUZA, E.D. de; COSTA, S.E.V.G. de A.; ANGHINONI, I.; CARVALHO, P.C. de F.; OLIVEIRA, E.V.F. de; MARTINS, A.P.; CAO, E.; ANDRIGHETTI, M. Soil aggregation in a crop-livestock integration system under no-tillage. Revista Brasileira de Ciência de Solo, v.34, p.1365-1374, 2010.

SULC, R.M.; TRACY, B.F. Integrated crop-livestock systems in the U.S. corn belt. Agronomy Journal, v.99, p.335-345, 2007.

TRAXLER, G.; FALCK-ZEPEDA, J.; ORTIZ-MONASTERIO R., J.I.; SAYRE, K. Production risk and the evolution of varietal technology. American Journal of Agricultural Economics, v.77, p.1-7, 1995.

VIANA, M.C.M.; ALBERNAZ, W.M.; PINTO JÚNIOR, E.S.; NOCE, M.A.; MENDES, M.A.; PORTUGAL, M.P.; ALVARENGA, R.C. Produção de milho e análise econômica do sistema de interagração lavoura-pecuária-floresta, em propriedades 
de agricultura familiar, na região central de Minas Gerais. In: CONGRESSO NACIONAL DE MILHO E SORGO, 28., 2010, Goiânia. Anais. Goiânia: Associação Brasileira de Milho e Sorgo, 2010. p.3404-3412.

VILELA, L.; MARTHA JÚNIOR, G.B.; MACEDO, M.C.M.; MARCHÃO, R.L.; GUIMARÃES JUNIOR, R.; PULROLNIK, K.; MACIEL, G.A. Integração lavoura-pecuária na região do Cerrado. Pesquisa Agropecuária Brasileira, v.46, p.?, 2011. (no prelo).

VILELA, L.; MIRANDA, J.C.C.; SHARMA, R.D.; AYARZA, M.A. Integração lavoura-pecuária: atividades desenvolvidas pela Embrapa Cerrados. Planaltina: Embrapa Cerrados, 1999. 31p. (Embrapa Cerrados. Documentos, 9).

WANDER, A.E.; SOUZA, R.D.S.; RICARDO, T.R.; SILVEIRA, P.M.D. Viabilidade econômica e risco da rotação e consorciação de cultivos para a integração lavoura-pastagem em condições irrigadas no Cerrado brasileiro. Informações Econômicas, v.40, p.34-42, 2010.

YOKOYAMA, L.P.; VIANA FILHO, A.; BALBINO, L.C.; OLIVEIRA, I.P. de; BARCELLOS, A. de O. Avaliação econômica de técnicas de recuperação de pastagens. Pesquisa Agropecuária Brasileira, v.34, p.1335-1345, 1999.

Recebido em 15 de abril de 2011 e aprovado em 30 de setembro de 2011 